\title{
Clinical phenotyping linked treatment strategies in patients with bladder pain syndrome
}

\begin{abstract}
Introduction: This is a clinically heterogeneous entity with suboptimal results to known modalities of treatment globally. The clinical heterogeneity of this clinical syndrome is due to variable etiopathogenetic mechanisms involved in the final manifestation of symptoms. Hence it is but logical to categorize these patients into phenotypes based on suspected underlying etiology, in order to institute a treatment strategy specific to that group.

Methodology: Diagnosed as BPS as per the ESSIC guidelines, 84 consecutive patients were categorized into four clinical phenotypes (CP), allergy, dysfunctional voiding, neuropathic pain and presence of Hunner's ulcer and labeled as CP 1 through 4 respectively. Patients could qualify to be categorized in more than one category. They were administered oral PPS and treatment specific to the CP. The patients from CP1 to 3 received hydroxyzine, clonazepam, and amitriptyline respectively. Patients with Hunners lesion underwent hydro distension and ablation of Hunners lesion followed by intravesical heparin and hydrocortisone solution. The patients were scored clinically and assessed as per prevailing scoring system and results were tabulated at 1,3 and 6 months.
\end{abstract}

Results: Out of 86 patients, 78 were found evaluable. The CP groups from 1-4 were found to have $24,46,14,18$ patients respectively. Many patients qualified for being categorized in more than one category. The response rate at 6 months was $71 \%, 76 \%, 57 \%$, and $78 \%$ respectively for each $\mathrm{CP}$ while the response of overall cohort was $76 \%$.

Conclusion: Clinical phenoptyping based on features indicative of etiology helps to improve outcomes by guiding treatments focused towards addressing the specific pathological processes.

Keywords: clibical phenotyping, bladder pain syndrome, interstitial cystitis

\author{
Volume 8 Issue 4 - 2020
}

\author{
Rajesh Taneja,' Deepak Kumar Chugh ${ }^{2}$ \\ 'Senior Consultant and Advisor, Urology and Robotic Surgery, \\ India \\ ${ }^{2}$ Clinical associate, Indraprastha Apollo Hospitals, India
}

Correspondence: Rajesh Taneja, M.Ch. Urology, Room Number 1019 (Gate number 10), Indraprastha Apollo Hospitals, New Delhi, India, I 10076, Tel +9| I I7|791019,

Fax+9III7I79I050, Email rajeshtanjadr@yahoo.com

Received: July 10, 2020 | Published: July 30, 2020

\section{Introduction}

Bladder Pain Syndrome (BPS) is a conglomeration of heterogeneous clinical entities ranging from intense trans mural inflammation with or without ulceration of urothelium of bladder, to pathological phenomena occurring elsewhere in body but manifesting through bladder as pain, urgency and frequency. Thus the syndrome encompasses patients suffering from urinary symptoms consequent to a variety of pathophysiological pathways working in bladder or non-bladder domains. ${ }^{1}$ It is therefore, expected that a single treatment can't be suited for all the patients as the etiology of individual patients is different. Etiopathogenesis of this heterogeneous entity is quite variable and if there could be a method of grouping or classifying these patients based on clinical clues to the underlying etiopathogensis, then therapies specific to addressing these respective pathogenic mechanisms may be used with good results. ${ }^{2}$ The objective of this study was to categorize patients of bladder pain syndrome on the basis of clinical clues towards the possible pathogenic process and apply specific modality for treatment of same.

\section{Aims and objectives}

To classify patients of Bladder Pain Syndrome (BPS) into 'Clinical Phenotypes' based on possible underlying predominant etiopathological process and apply specific modality of treatment.

\section{Methodology}

During February 2014 and March 2019, 84 Consecutive patients diagnosed as Bladder Pain Syndrome (BPS) as per ESSIC guidelines were included in the study. ${ }^{3}$ They were informed about the fact that the treatment being administered was focused on the features, which qualify them as a special group within the patients of BPS. They were also informed that no new treatment modality was being instituted per se. All patients underwent cystoscopy under general anesthesia. The patients were classified into four Clinical Phenotypes (CP) based upon the clinical indicators.

\section{CP I allergy}

Patients with history of any kind of allergies are included in this group.
I. Seasonal rhinitis,
II. Vasomotor rhinitis
III. Asthma,
IV. Urticaria and Hives
V. Dermatological hypersensitivity
VI. Allergies to food items
VII. Allergies to drugs

VIII. Any other history suggestive of allergies

\section{CP 2 Dysfunctional voiding}

Patients having 3 or more of following features were included in this category. 
I. Marked hesitancy especially difficulty in initiating stream in public

II. Nocturia $<3$

III. Constipation

IV. Aching Pain in anal canal

V. Clinical signs of overactive pelvic floor

VI. Presence of Myofacial bands in perineal muscles

\section{CP 3 Neuropathic pain}

Patients having any one of following

I. Pricking pain in vulva commonly clitoris, forcing them to void

II. Burning pain in perineum

III. Burning pain in anus somewhat relieved on passing urine.

\section{CP 4 Hunners lesions}

a) Hunner's lesions on cystoscopy

Many patients qualified to be placed in more than one group. The $\mathrm{CP}$ specific treatments that they received are as given below.

\section{CP I Allergy}

Hydroxyzine $(25 \mathrm{mg})$ once a day.

\section{CP 2 Dysfunctional voiding}

Skeletal muscle relaxants (Clonazepam $0.25 \mathrm{mg}$ twice a day), Pelvic floor relaxation exercises by a specially tailored program supervised by physiotherapist.

\section{CP 3 Neuropathic pain}

Amitriptyline $25 \mathrm{mg}$ at bedtime.

\section{CP 4 Hunner's lesion}

Hydrodistension, cystoscopic ablation followed intravesical instillation of Heparin and hydrocortisone solution once a week for six weeks. ${ }^{4}$ Since many patients qualified for being grouped in more than one $\mathrm{CP}$, the treatment specific for these was administered. In addition, all patients received Oral Pentosan Polysulphate (PPS) at
$100 \mathrm{mg}$ three times a day through out the study period. Patients with minimal follow up of 6 months were included in the study.

The patients' symptoms were scored according to the prevalent clinical scoring system in the institution at the time of induction at 1 , 3 and 6 months. ${ }^{5}$ The score was plotted on the scale from $0-50$ points. Patients' response was stratified into three categories.

\section{Stratification of response}

\section{Unsatisfactory response}

Little or no relief in pain despite 6 months of treatment (Drop in score less than 10).

\section{Good response}

Significant relief of pain with manageable reduction/without significant reduction in frequency (Drop in score between 11 and 29).

\section{Excellent response}

Relief of pain as well as significant reduction in frequency (drop in score more than 30 ). The clinical scores were tabulated and analyzed.

\section{Observations and results}

Of the 86 patients, 78 qualified for the study, as the rest did not complete the minimum follow up period of 6 months. Of these patients, the various CP groups from 1-4 had 24,46,14,18 patients respectively. As mentioned earlier, many patients qualified to be placed in more than one CP group. Of the 24 patients in CP 1(Allergy) group, $17 \%, 8 \%$ and $50 \%$ had dysfunctional voiding, neuropathic pain and Hunner's lesions respectively. Analysis of patients in CP 2(Dysfunctional Voiding) group revealed that $17 \%, 9 \%$ and $2 \%$ patients also had neuropathic pain, allergy and Hunner's lesion respectively (Figure 1). The patients of CP 3 (Neuropathic pain) group had overlapping features of dysfunctional voiding in 57\%, allergy in $14 \%$, Hunner's Lesion in 7\%. Sixty seven percent of patients of CP 4 (Hunner's Lesion) group had additional features of allergy while 5.5 $\%$ had dysfunctional voiding and a similar percentage had neuropathic pain. The CP specific feature has been tabulated as $100 \%$ in each group as that was the defining criteria of the CP. These have been depicted in Table 1. Phenotype group specific and over all response as per the clinical scoring at 1,3 and 6 months has been has been tabulated in Table 2 .

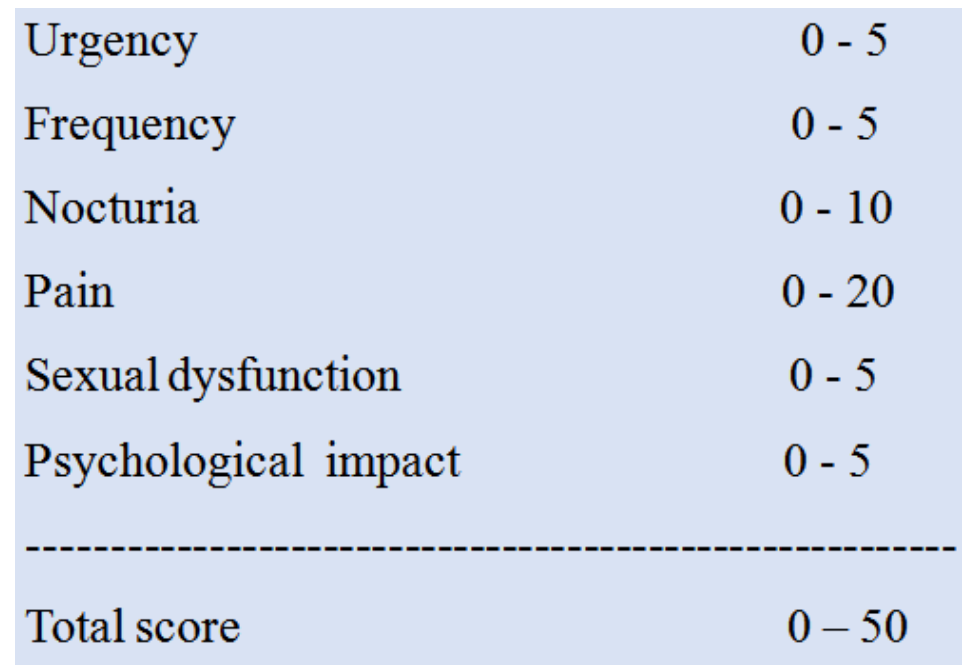




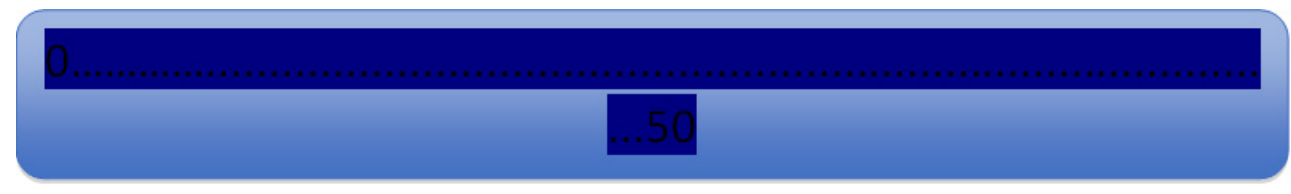

BPS Symptom scale.

Figure I Clinical Scoring System and Scale. ${ }^{5}$

Table I Associated features in patients classified in each Clinical Phenotype (CP)

\begin{tabular}{lllll}
\hline & $\begin{array}{l}\text { CPI Allergy } \\
(\mathbf{n = 2 4 )}\end{array}$ & $\begin{array}{l}\text { CP2 - Dysfunctional } \\
\text { voiding (n=46) }\end{array}$ & $\begin{array}{l}\text { CP3 Neuropathic } \\
\text { pain(n=14) }\end{array}$ & $\begin{array}{l}\text { CP4 - Hunner's } \\
\text { lesion (n= I 8) }\end{array}$ \\
\hline Associated feature & & & & $67 \%$ \\
\hline Allergy & $100 \%$ & $9 \%$ & $14 \%$ & $5.50 \%$ \\
Dysfunctional voiding & $17 \%$ & $100 \%$ & $57 \%$ & $5.50 \%$ \\
Neuropathic pain & $8 \%$ & $17 \%$ & $100 \%$ & $100 \%$ \\
Hunners Lesion & $50 \%$ & $2 \%$ & $7 \%$ & $21 \%$ \\
None other & $25 \%$ & $72 \%$ & $22 \%$ & \\
\hline
\end{tabular}

Table 2 Response to treatment during follow up

\begin{tabular}{|c|c|c|c|c|c|}
\hline & CPI Allergy & $\begin{array}{l}\text { CP2 Dysfunctional } \\
\text { voiding }\end{array}$ & $\begin{array}{l}\text { CP3 Neuropathic } \\
\text { pain }\end{array}$ & $\begin{array}{l}\text { CP4 Hunner's } \\
\text { lesion }\end{array}$ & $\begin{array}{l}\text { Overall } \\
\text { cohort }\end{array}$ \\
\hline \multicolumn{6}{|c|}{ Response in percentage } \\
\hline \multicolumn{6}{|c|}{ At I month } \\
\hline Excellent & $33 \%$ & $57 \%$ & $50 \%$ & $89 \%$ & $67 \%$ \\
\hline Good & $50 \%$ & $26 \%$ & $14 \%$ & $11 \%$ & $15 \%$ \\
\hline Poor & $15 \%$ & $17 \%$ & $36 \%$ & $0 \%$ & $18 \%$ \\
\hline \multicolumn{6}{|c|}{ At 3 Months } \\
\hline Excellent & $67 \%$ & $34 \%$ & $57 \%$ & $83 \%$ & $71 \%$ \\
\hline Good & $25 \%$ & $33 \%$ & $22 \%$ & $17 \%$ & $14 \%$ \\
\hline Poor & $8 \%$ & $33 \%$ & $21 \%$ & $0 \%$ & $15 \%$ \\
\hline \multicolumn{6}{|c|}{ At 6 months } \\
\hline Excellent & $71 \%$ & $76 \%$ & $57 \%$ & $78 \%$ & $76 \%$ \\
\hline Good & $21 \%$ & $7 \%$ & $29 \%$ & $22 \%$ & $10 \%$ \\
\hline Poor & $8 \%$ & $17 \%$ & $21 \%$ & $0 \%$ & $14 \%$ \\
\hline
\end{tabular}

\section{Discussion}

In the current study, patients, who were classified based on predominant features of a defined $\mathrm{CP}$, had overlying features in many cases. However 46/78 (59\%) did not have any overlapping features. It is interesting to note that the largest percentage of such patients was from the CP 2 (dysfunctional voiding) group (72\%). There was a bidirectional association of presence of allergy with the Hunner's lesion as $50 \%$ of patients in CP 1 (allergy) had Hunner's lesion and $67 \%$ of patients with CP 4 (Hunner's lesion) had allergy. This may point towards the bladder centric inflammatory process working in these two groups. While $57 \%$ of CP 3 (neuropathic pain) also had dysfunctional voiding, only $17 \%$ of CP 2 (dysfunctional voiding) had neuropathic pain. Both these groups had a lower incidence of Hunner's lesion ( $2 \%$ and $7 \%$ respectively). These observations point towards etiological pathways working outside the bladder and urinary symptoms and bladder pain resulting seem to be a secondary manifestation of extra vesical pathology. Clinical phenotyping based on symptoms has been attempted by Nickel et al and a system of UPOINT has been evaluated. ${ }^{6}$ Others have also tried to classify the patients into groups but these appear to be based on clinical manifestation rather than based on possible etiopathogensis. ${ }^{7,8}$ It has been proposed that the patients with Hunner's lesion should be treated as a separate disease entity. ${ }^{9}$ The current study aims at classifying patients on the basis of possible etiopathogensis and administering suitable treatment.

The CP 1 or the allergy group is straightforward in definition as mentioned in methodology. The final pathway of injury to bladder manifesting as inflammation is supposed to be through mast cell 
activation. Mast cells are also important pathways in manifestation of allergies in various parts of body. So it is reasonable to administer treatment aimed at the final pathway through blocking these effects by the use of hydroxyzine. Theorhides and Sant recognized this fact and published an open-ended study in 1997, depicting the role of hydroxyzine in treatment of interstitial cystitis patients with history of allergies and mastocytosis of bladder. ${ }^{10}$ Hydroxyzine is a competitive inhibitor of $\mathrm{H}_{1}$ receptors of histamine and blocks the final pathway of inflammation arising out of allergic mechanism thus blocking pruritus, urticarial reactions etc. non specific Histamine antagonist. The CNS effect causes sedation and appears to be dose related suppression of subcortical activity. It has mild anticholinergic effects resulting in relaxation of smooth and skeletal muscles. It also has mild analgesic properties. Sedation appears to be a limiting side effect of this drug.

The patients in CP 2 had history of difficulty in initiating the stream of urine, had marked hesitancy, but conspicuously nocturia limited to 2-3 per night. On clinical examination there were signs of overactive pelvic floor, or myo- fascial bands in the pelvic floor as felt per vaginally. Usually the pain would also be felt in anus. Superficial dyspareunia and constipation were also considered as pointers towards this category. These patients had painful urgency and frequency when awake. Once asleep, their symptoms were minimal. These patients also had exacerbations in stressful situations and relief in periods of relative ease and enjoyment. These patients also reported relief after a session of pelvic floor relaxation physiotherapy or a bout of swimming. These patients seemed to benefit from the use of skeletal muscle relaxants like clonazepam, cyclobenzaprine or baclofen. These further confirm the occurrence of hyperactive pelvic floor in these patients. The presence of hyperactive pelvic floor in patients with BPS has been known to occur up to $85 \%$ in various studies. ${ }^{11}$ It has been postulated that hyperactive pelvic floor could result in turbulence during voiding and in some cases resultant inflammation of trigone. However this is not expected to cause any extensive mucosal inflammation of the bladder, hence the absence of stigmata of inflammation on cystoscopy. Usually the bladder capacity is adequate under anesthesia and these patients may not benefit from or even require hydro distension.

In the current series, patients classified under CP 3 (neuropathic pain) reported pricking or burning pain in the vulva, especially clitoris, perineum and anal canal. This is usually intensified on filling of bladder, forcing the patients to void. The pain could also radiate to perineum and inner thigh. The focussed neurological examination on these patients is must to exclude any identifiable neurological cause like weakness of muscles or sensory neuropathy. If the doubt persists, suitable nerve conduction studies may be undertaken to exclude any organic neural pathology. Presence of cystoscopic stigmata of bladder inflammation were unusual in these patients. The neuronal nature of pain i.e. burning or pricking sensation, points towards some kind of neurogenic pathophysiology/inflammation. These patients were found to respond to addition of amitriptyline to their regimen. Amitriptyline is a tricyclic antidepressant with a specific role in neurogenic pain has been studied in past for its use in BPS. ${ }^{12}$ However these studies have used it without the segregation of BPS patients as followed in the current study, thereby and understandably so, showing suboptimal results. The main side effect of amitriptyline is sedation, which needs to be warned against. The dose to be started is $10 \mathrm{mg}$ at bedtime. Many patients do not report any sedation at this dose and this could be escalated to $25 \mathrm{mg}$ at bedtime. The effect of amitriptyline in some patients is a function of time and the beneficial effects improve with prolonged duration of treatment. This has been observed and documented in published literature. ${ }^{13}$ Clinical segregation and specific use of amitriptyline in such patients with clinical indicators towards neurogenic inflammation improves the over all result in patients of BPS.

Patients with Hunner's lesions (CP 4) have intense pain in lower abdomen and this pain is somewhat relieved on passing urine. The frequency may be intense, sometimes between 30-45 minutes, without any diurnal variation. The voided volume is usually small, less than $200 \mathrm{ml}$. Usually; there is deep dyspareunia in these women. Suprapubic tenderness is often present in most of these patients. In male patients, absence of tenderness over prostate on digital rectal examination but presence of suprapubic tenderness can differentiate BPS from chronic prostatitis. ${ }^{14}$ In many patients, the pain could be reported towards one side of hypogastrium, and this could also be correlated with the presence of Hunner's lesion on that side of bladder. In past other authors have attempted to clinically predict the presence of Hunner's lesion but have not been successful in that and have suggested the importance of cystoscopy. ${ }^{15}$ Cystoscopy, hydro distension and ablation of Hunner's lesion are almost always effective in alleviating symptoms, at least for a variable period soon after the procedure. ${ }^{16}$ More often than not the pain is almost gone. As per the protocol these patients then receive weekly intra vesical instillations of Hydrocortisone $(200 \mathrm{mg}$ ) and Heparin 25000 (IU) in a physiological saline adding up to a total of $40 \mathrm{ml}$ solution for next six weeks, starting soon after the procedure. ${ }^{4}$ The effect of hydro distension appears to last for few months and many of these patients require getting a repeat hydro distension and ablation of any recurrent Hunner's lesions. This has also been documented by other researchers. ${ }^{17}$ None of our patients required undergoing a repeat therapy within 6 months period. The results of individual $\mathrm{CP}$ groups and the overall cohort appear to be better than the usual reported rates in such population of patients. This is an indicator of the fact that etiological grouping is likely to help administration of specific treatment modalities. It has been indicated by others that etiology-based phenotyping should be more appropriate. ${ }^{18}$ The prognosis of individual CP may be predictable if this strategy is used for long term in multiple centres and data is collated and analysed together.

\section{Conclusion}

Segregation of BPS patients by clinical phenotyping based on possible indicators of etio-pathogenetic mechanisms seems to help improve the treatment outcome if specific modalities of treatment are administered. Clinical phenotyping in this manner may also be used to predict the outcome in a given patient in future.

\section{Acknowledgments}

None.

\section{Disclosures}

The authors have no competing interest to disclose.

\section{References}

1. Hanno PM, Nordling J, Staskin DR, et al. Bladder Pain syndrome- an Evolution. Springer USA. 2018.

2. Akiyama Y, Luo Y, Hanno PM, et al. Interstitial cystitis/bladder pain syndrome: The evolving landscape, animal models and future perspectives. Int J Urol. 2020;27(6):491-503.

3. van de Merwe JP, Nordling J, Bouchelouche P, et al. Diagnostic criteria, classification, and nomenclature for painful bladder syndrome/interstitial cystitis: an ESSIC proposal. Eur Urol. 2008;53(1):60-67. 
4. Taneja R, Jawade K. A Rational combination of intravesical and systemic agents in the treatment of interstitial cystitis. Scandinavian journal of nephrology and urology. Journal Scandinavian Journal of Urology and Nephrology. 2007;41(6):511-515.

5. Taneja R, Massand S. A Modified clinical scoring system for Bladder Pain Syndrome: Long term experience. International Journal of Urology. 2019;26(Suppl.1):61-67.

6. Nickel JC1, Shoskes D, Irvine-Bird K. Clinical phenotyping of women with interstitial cystitis/painful bladder syndrome: a key to classification and potentially improved management. J Urol. 2009;182(1):155-160.

7. Kartha GK1, Kerr H, Shoskes DA. Clinical phenotyping of urologic pain patients. Curr Opin Urol. 2013;23(6):560-564.

8. Acar Ö, Tarcan T. Cystoscopic evaluation and clinical phenotyping in interstitial cystitis/bladder pain syndrome. $J$ Turk Ger Gynecol Assoc. 2019;20(2):117-122.

9. Magnus Fall, Jørgen Nordling, Mauro Cervigni, et al. Hunner lesion disease differs in diagnosis, treatment and outcome from bladder pain syndrome: an ESSIC working group report. Scandinavian Journal of Urology. 2020;54(2):91-98.

10. Theoharides TC, Sant GR. Hydroxyzine therapy for interstitial cystitis. Urology. 1997;49(5A Suppl):108-110.

11. Gupta P, Gaines N, Sirls LT, et al. A multidisciplinary approach to the evaluation and management of interstitial cystitis/bladder pain syndrome: an ideal model of care. Transl Androl Urol. 2015;4(6):611-619.
12. Hanno PM, Burks DA, Clemens Q. American Urological Association (AUA) guideline. Diagnosis and treatment of interstitial cystitis/bladder pain syndrome. Amended 2014.

13. Van Ophoven A, Pokupic S, Heinecke A, et al. A prospective, randomized, placebo controlled, double-blind study of amitriptyline for the treatment of interstitial cystitis. J Urol. 2004;172(2):533-536.

14. Shoskes DA, Nickel JC, Rackley RR, et al. Clinical phenotyping in chronic prostatitis/chronic pelvic pain syndrome and interstitial cystitis: a management strategy for urologic chronic pelvic pain syndromes. Prostate cancer Prostatic Dis. 2009;12(20):177-183.

15. Doiron RC, Tolls V, Irvine-Bird K, et al. Clinical Phenotyping Does Not Differentiate Hunner Lesion Subtype of Interstitial Cystitis/Bladder Pain Syndrome: A Relook at the Role of Cystoscopy. Urol. 2016;196(4):11361140

16. Malykhina A1, Hanno P. How are we going to make progress treating bladder pain syndrome? ICI-RS 2013. Neurourol Urodyn. 2014;33(5):625-659.

17. Lai HH, Pickersgill NA, Vetter JM. Phenotype in Interstitial Cystitis/ Bladder Pain Syndrome: A Systematic Review and Meta-Analysis. J Urol. 2020 .

18. Ogawa $\mathrm{T}$, Ishizuka $\mathrm{O}$, Ueda $\mathrm{T}$, et al. Current and emerging drugs for interstitial cystitis/bladder pain syndrome (IC/BPS). UGS 2015;20(4):555-570. 